\title{
Influence of Different Modified Gears on the Vibration and Noise of Electric Vehicle Reducer
}

\author{
Bo Xu \\ School of Automotive and Traffic Engineering, Jiangsu University, Zhenjiang 212013, China. \\ Yu Qiu \\ School of Mechanical Engineering, Jiangsu University, Zhenjiang 212013, China.

\begin{abstract}
Bifeng Yin
School of Automotive and Traffic Engineering, Jiangsu University, Zhenjiang 212013, China. E-mail: ybf@ujs.edu.cn
\end{abstract}

\author{
Xijun Hua and Hang Du \\ School of Mechanical Engineering, Jiangsu University, Zhenjiang 212013, China.
}

\begin{abstract}
(Received 10 August 2021; accepted 24 October 2021)
The tooth profile crowning modification was applied onto paired gears for reducing the noise of an electric vehicle reducer. The simulated gear contact spots are compared before and after modification, and are validated by a contact spot experiment. Based on the rigid-flexible coupling model of the gear transmission system, the timevarying meshing stiffness, time-varying meshing force, and the vibration and noise of the gear pairs with different modified gears obtained by simulating calculation are analyzed. The results showed that the selection of modified gear has a great influence on the modification effect. In the way of tooth profile crowning, it is not advisable to modify the pinion independently, as it may increase the frequency and degree of meshing impact between the helical gear pair, making the transmission become less smooth; while modifying the wheel and pinion at the same time can effectively reduce the time-varying meshing stiffness and force, and the vibration and noise. Also, the optimized gear modification scheme is verified by the noise test.
\end{abstract}

\section{INTRODUCTION}

In recent years, with the continuous development of the electric vehicle industry, the performance of an electric vehicle's transmission system and it's driving speed is constantly rising. However, due to the absence of an engine, the vibration and noise of the electric vehicle, which mainly come from the gearbox and the reducer, would be intuitively felt by the occupants. The reducer is used to reduce the speed and increase the torque, which plays a key role in the driving process of electric vehicles. For commercial electric vehicles, especially private cars, the gear noise of the reducer is obvious. Generally, the vibration and noise of the gear are caused by the inaccurate transmission of the gear. To a large extent, the existence of noise also damages the performance of the equipment and reduces the service life. Therefore, it is of great significance to put forward the requirements of lower noise, vibration and harshness $(\mathrm{NVH})$ for the gear of electric vehicle reducer.

On the issue of vibration and noise reduction of gear boxes, a large number of scholars have provided valuable ideas and theoretical support. From the perspective of gear modification, Tanaka. et al., used the electrical resistance method to measure the duration of contact point elastic dynamic lubrication (EHL) condition, and studied the effect of tooth profile modification on the durability of the stimulating gear surface. ${ }^{1}$ Fatourehchi et al. studied the tooth profile modification impact on planetary gears, and they established a systematic modification method including tribology and subsurface stress analysis to ensure the high durability of the modified gear and achieve better transmission efficiency. ${ }^{2}$ Wang combined tooth surface contact analysis (TCA) and loaded tooth surface contact analysis (LTCA) to simulate the meshing process of gears, and proposed a three-dimensional repair method to modify gears. ${ }^{3}$ Yang et al. deduced the tooth profile modification equation and established the translation-torsion nonlinear dynamic model in response to the requirement of reducing gear meshing impact. ${ }^{4}$ According to the principle of parabolic transmission error, Samani et al. proposed a new nonlinear vibration model the higher-order transmission error (THE) method to reduce the vibration of spiral bevel gears. ${ }^{5}$ Ren et al. established a multi-degree-of-freedom nonlinear dynamic model of the cycloid reducer. The Runge-Kutta numerical method was used to solve the relationship between the rotation displacement and the rotation speed of the cycloid disc with different displacement gaps. ${ }^{6}$ Brethee et al. considered the influence of friction on the dynamic response of gear system. ${ }^{7}$

Existing literature points that transmission error is one of the main excitations that cause gear vibration and noise. Hjelm et al. studied the relationship between transmission error, contact pressure and manufacturing error tolerance. ${ }^{8}$ Wang. et al., investigated the influence of gear thermal deformation on transmission error. ${ }^{9}$ Duan et al. introduced the flexible hous- 
ing into dynamic analysis, and they found that appropriately reducing the stiffness of the shell can reduce the transmission error by carrying out experimental verification. ${ }^{10}$ Slamani et al. used a laser interferometer system to measure gear transmission error. ${ }^{11}$ Wink. et al., considered the influence of gear tooth contact deviation and proposed an accurate calculation method for gear transmission error. ${ }^{12}$ Palermo et al. used digital encoders to study the transmission error of electric vehicle gearbox gears. In addition, gear mesh stiffness is another main excitation. ${ }^{13}$ Based on the potential energy method, Elyousfi et al. calculated the mesh stiffness of gear with surface defects with considering the variation of defect depth in width and length directions. ${ }^{14}$ Dai et al. thought the time-varying meshing stiffness is the main excitation source of gear vibration, and compared the time-varying meshing stiffness under different torques. ${ }^{15}$ Chen et al. calculated the gear stiffness by taking the complex foundation type and crack propagation path into consideration, and found the tooth crack and rim fracture have larger effects on the meshing stiffness of single-tooth contact area in comparison with the double-tooth contact area. ${ }^{16}$ Raghuwanshi et al. proposed a new experimental technique based on experimental mode analysis (EMA) to measure the mesh stiffness of gear pairs. ${ }^{17}$ Chen et al. proposed a comprehensive analysis and calculation model of gear meshing stiffness and transmission error excitation. ${ }^{18}$

The finite element method (FEM) simulation is the main method for solving engineering problems that involve gear transmission. Tang et al. took the electric multiple units (EMU) traction helical gear as an object, by modeling the three-dimensional helical gear in ANSYS software, the contact of gears under starting and high-speed working conditions was analyzed. ${ }^{19}$ Liu et al. constructed a parameterized solid model of the gear and gearbox, and analyzed the dynamic performance of the gear system. ${ }^{20} \mathrm{Jia}$ et al. established a dynamic model of casing transmission, and obtained the actual meshing state of each gear pair through dynamic simulation. ${ }^{21}$ Zhang et al. establish a complex three-dimensional gear meshing model in Romax to calculate the contact pressure and transmission error. ${ }^{22}$ Fang et al. considered the influence of elastic deformation in the dynamic modeling, and established a rigid-flexible coupling dynamic model. ${ }^{23}$ To study the causes of gear vibration, Mark decomposed the transmission error spectrum of meshing gear pair into the harmonics generated by the basic period of gear pair, the rotation harmonics and the tooth meshing harmonics of single gear. ${ }^{24}$

In this research, based on the tooth profile crowning modification, the gear pair of an electric vehicle reducer was modified with three different schemes, and the effects of modification were compared by contact spot simulation and experiment. After establishing the rigid-flexible coupling model of the gear transmission system, the time-varying meshing stiffness and force under different modification modes were analyzed. Also, the sound power was obtained by using the acoustic simulation at different speeds, followed by the validation of noise bench test, so as to clarify tooth profile crowning modification effects on different gears according to the working conditions, which could provide guidance for the gear selection and modification in engineering application.
Table 1. Unmodified gear parameters.

\begin{tabular}{||c|c|c|}
\hline Parameter & Pinion & Wheel \\
\hline Modulus $(\mathrm{mm})$ & 2.25 & 2.25 \\
\hline Face width $(\mathrm{mm})$ & 28 & 30 \\
\hline Number of teeth & 30 & 51 \\
\hline Profile shift coefficient & 0.1136 & -0.1340 \\
\hline Pressure angle $\left(^{\circ}\right)$ & 17.5 & 17.5 \\
\hline Helix angle $\left(^{\circ}\right)$ & 32.5 & 32.5 \\
\hline Transverse contact ratio & 1.537 & 1.537 \\
\hline Axial contact ratio & 2.057 & 2.057 \\
\hline Backlash $(\mathrm{mm})$ & 0.05 & 0.05 \\
\hline Material & $20 \mathrm{MnCr} 5$ & $20 \mathrm{MnCr} 5$ \\
\hline
\end{tabular}

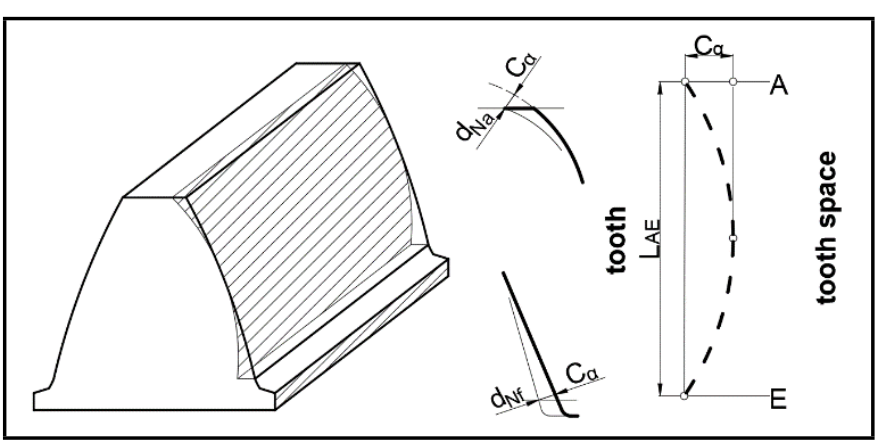

Figure 1. Diagram of tooth profile crowning modification.

\section{SYSTEM DESIGN}

\subsection{Gear Modification}

Aiming at the gear knocking phenomenon in electric vehicle reducer under different speeds, a pair of helical cylindrical gears of a reducer was selected as the research object, and the gear parameters are shown in Table 1.

The method of gear modification involves the removal of the "interference" part of the gear pair in the unideal meshing state, to reduce the meshing impact and load fluctuation degree of the gear pair, and to avoid the violent gear vibration. Generally, the crowning shape modification scheme has been adopted in the industry. Here we adopted the tooth profile crowning modification, the basic principles and parameters are shown in Figure 1. The purpose was to reduce the thickness of the top and root of the gear and modify the tooth profile to make the middle of the gear convex, which was different from linear modification and entire involute modification. The modification accuracy of the tooth profile crowning could be controlled within $1 \mu \mathrm{m}$ by grinding or ultra-finishing, and the processing accuracy should be strictly guaranteed in the processing process.

In Figure $1, d_{N a}$ and $d_{N f}$ were usable tip diameter and active root diameter. $A$ and $E$ were tooth tip and tooth root support points respectively. $L_{A E}$ was unwound tooth depth length. $C_{\alpha}$ was the amount of tooth tip tooth root modification. In this article, the specific value of $C_{\alpha}$ was the modification amount of pinion and wheel. Under the condition of tooth profile crowning modification, it is necessary to compare the influence of different gear modification. This research fully considered the three possibilities of simultaneous modification of wheel and pinion, modifying pinion independently, and modifying wheel independently. The schemes are shown in Table 2, and the modification quantity was obtained according to the simulation modification result of KISSsoft. To be specific, the modification quantity of the gear pair was selected when the Hertz 


Table 2. Modification schemes.
\begin{tabular}{|c|c|c|c|}
\hline \hline Program & a & b & c \\
\hline Shape modification & Profile crowning & Profile crowning & Profile crowning \\
\hline Modification gear & Wheel \& Pinion & Wheel & Pinion \\
\hline Modified parts & Addendum root & Addendum root & Addendum root \\
\hline Pinion $(\mu m)$ & 3 & 0 & 3 \\
\hline Wheel $(\mu m)$ & 3 & 3 & 0 \\
\hline
\end{tabular}

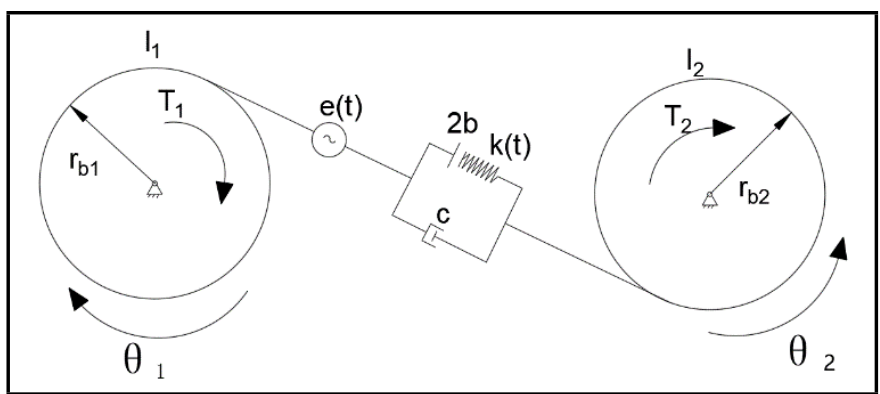

Figure 2. Non-linear mathematical model of gear transmission.

contact stress and transfer error reach the minimum value at the same time under this working condition.

\subsection{Mathematical Model of Nonlinear Vibration of Gear Pair}

According to the torsion principle of degree of freedom of parallel shaft gear, the nonlinear vibration mathematical model of helical gear pair was established. Under the ideal condition that all components in the reduction gearbox except the gear are regarded as absolute rigid bodies without considering any error, a pair of involute helical gear meshing pairs were simplified to the nonlinear mathematical model shown in Figure 2.

The system kinetic energy was given by:

$$
T=\frac{1}{2}\left(I_{1} \dot{\theta}_{1}^{2}+I_{2} \dot{\theta_{2}^{2}}\right) .
$$

The system potential energy was given by:

$$
U=\frac{1}{2} k(t) \delta^{2}
$$

The system dissipated energy was given by:

$$
R=\frac{1}{2} c \dot{\delta^{2}} .
$$

The deformation of the gear pair $\theta_{z}$ direction vibration projected to the direction of the meshing line was given by:

$$
\delta=\left(r_{b 1} \theta_{1}-r_{b 2} \theta_{2}\right) \cos \beta+e(t) .
$$

Then the total deformation of the system vibration from all directions projected to the direction of the meshing line was given by:

$$
\begin{aligned}
& \delta=\left(r_{b 1} \theta_{1}-r_{b 2} \theta_{2}\right) \cos \beta+e(t) ; \\
& \delta=\left(r_{b 1} \dot{\theta_{1}}-r_{b 2} \dot{\theta_{2}}\right) \cos \beta+\dot{e}(t) .
\end{aligned}
$$

The system incentives included the moment generated in the $\theta_{1}$ direction and $\theta_{2}$ direction given by:

$$
Q_{\theta_{1}}=+T_{1}
$$

$$
Q_{\theta_{2}}=-T_{2} \text {. }
$$

According to the Lagrangian energy equation:

$$
\begin{gathered}
T=\frac{1}{2}\left(I_{1} \dot{\theta_{1}^{2}}+I_{2} \dot{\theta_{2}^{2}}\right) \\
U=\frac{1}{2} k(t)\left[\left(r_{b 1} \theta_{1}-r_{b 2} \theta_{2}\right) \cos \beta+e(t)\right]^{2} ; \\
R=\frac{1}{2} c\left[\left(r_{b 1} \dot{\theta_{1}}-r_{b 2} \dot{\theta_{2}}\right) \cos \beta+\dot{e}(t)\right]^{2} ; \\
F_{m}=k(t) e(t)+c \dot{e}(t) .
\end{gathered}
$$

The system dynamics equation was obtained as:

$$
\begin{aligned}
& I_{1} \ddot{\theta}_{1}+k(t) r_{1} \cos \beta^{2}\left(r_{b 1} \theta_{1}-r_{b 2} \theta_{2}\right) \\
& \quad+c r_{1} \cos \beta^{2}\left(r_{b 1} \dot{\theta_{1}}-r_{b 2} \dot{\theta_{2}}\right)=T_{P}-F_{m} r_{1} \cos \beta ;
\end{aligned}
$$

$$
\begin{aligned}
& I_{2} \ddot{\theta}_{2}-k(t) r_{2} \cos \beta\left(r_{b 1} \theta_{1}-r_{b 2} \theta_{2}\right) \\
& \quad-c r_{2} \cos \beta\left(r_{b 1} \dot{\theta_{1}}-r_{b 2} \dot{\theta_{2}}\right)=-T_{P}+F_{m} r_{2} \cos \beta ;
\end{aligned}
$$

where $T_{1}$ was the gear input torque, $T_{2}$ was the load torque, $r_{2}$ was the output gear base circle radius, $r_{1}$ was the input gear base circle radius, $I_{2}$ was the moment of inertia of the output gear around the z-axis, and $I_{1}$ was the moment of inertia of the input gear around the $\mathrm{z}$-axis , $\theta_{2}$ was the torsional vibration displacement of the output gear around the z-axis, $\theta_{1}$ was the torsional vibration displacement of the input gear around the z-axis, $e(t)$ was the transmission error of the gear pair, $k(t)$ was the meshing stiffness, $c$ was the meshing damping, and $\alpha$ was Pressure angle, $\beta$ was the helix angle of the base circle.

\subsection{Gear Mesh Stiffness}

Gear meshing stiffness refers to the comprehensive effect of each pair of gears participating in the meshing in the entire meshing area, which is mainly related to the elastic deformation of a single tooth, the comprehensive elastic deformation of a pair of gears and the coincidence degree of the gears. The LMS Virtual. Lab provides two different methods for calculating the time-varying meshing stiffness. In this paper, the ISO method was selected to take the influence of gear tooth width and coincidence into consideration. The ISO $6336-1: 2006$ standard stipulated that the average value of meshing stiffness of a single tooth was given by:

$$
\begin{gathered}
c^{\prime}=c^{\prime}{ }_{t} h C_{M} C_{R} C_{B} \cos \beta \\
c^{\prime}{ }_{t} h=\frac{1}{q^{\prime}}
\end{gathered}
$$

$$
\begin{aligned}
q^{\prime}=C_{1}+\frac{C_{2}}{z_{n 1}}+ & \frac{C_{3}}{z_{n 2}}+C_{4} x_{1}+\frac{C_{5} x_{1}}{z_{n 1}} \\
& +C_{6} x_{2}+\frac{C_{7} x_{2}}{z_{n 2}}+C_{8} x_{1}^{2}+C_{9} x_{2}^{2}
\end{aligned}
$$

$$
\begin{gathered}
C_{B}=\left[1.0+0.5\left(1.2-\frac{h_{f p}}{m_{n}}\right)\right]\left[1.0-0.02\left(20^{\circ}-\alpha_{p n}\right)\right] ; \\
C_{M}=0.8, C_{R}=1.0 ;
\end{gathered}
$$




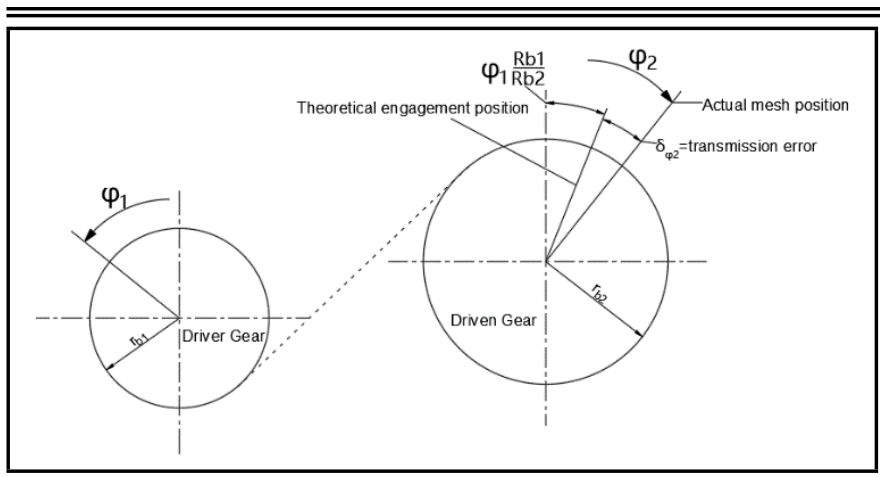

Figure 3. Diagram of gear transmission error .

in the formula, $c_{t h}^{\prime}$ was the theoretical value of the stiffness of a single pair of teeth; $C_{M}$ was the theoretical correction coefficient; $C_{R}$ was the spoke coefficient; $C_{B}$ was the basic rack coefficient; $\beta$ was the helix angle. $h_{f p}$ was the tooth root height of the basic rack tool of the cylindrical gear; $m_{n}$ was the normal modulus; $\alpha_{p n}$ was the normal pressure angle of the basic rack tool of the cylindrical gear.

\subsection{Gear Transmission Error}

Gear transmission error refers to the error between the actual meshing position of the gear pair and the ideal position under the condition of ideal gear meshing.

In the Fig. 3, $r_{b 1}$ and $r_{b 2}$ were the base circle radii of the driving gear and the driven gear respectively, $\varphi_{1}$ and $\varphi_{2}$ were the actual rotation angles of the driving gear and the driven gear respectively. The transmission error $\delta \varphi_{2}$ was defined as:

$$
\delta \varphi_{2}=\varphi_{2}-\varphi_{1} \frac{r_{b 1}}{r_{b 2}}
$$

The transmission error calculation based on theoretical gear tooth contact analysis was mainly to establish the mathematical contact model of the meshing gear pair, calculate the transmission error of the gear system by solving the relationship between the rotation angle of the driven gear and the rotation angle of the driving gear according to the continuous contact conditions. The calculation process was as follows:

1. Establish the fixed coordinate systems $S_{1}, S_{2}$ and $S_{f}$ for gear 1 , gear 2 and the frame respectively, and add a fixed coordinate system $S_{q}$ to simulate the installation error. 2. Establish the tooth surface equation. The two tooth surfaces $\Sigma_{1}$ and $\Sigma_{2}$ are expressed in the respective gear coordinate systems $S_{1}$ and $S_{2}$ as:

$$
\delta \varphi_{2}=\varphi_{2}-\varphi_{1} \frac{r_{b 1}}{r_{b 2}}
$$

in the formula, $r_{i}$ was the tooth surface position vector equation; $u_{i}, \theta_{i}$ were the tooth surface processing parameters.

The unit normal vector of the surface was expressed as:

$$
n_{i}=\frac{\frac{\partial r}{\partial u_{i}} \times \frac{\partial r_{i}}{\partial \theta_{i}}}{\left|\frac{\partial r_{i}}{\partial u_{i}} \times \frac{\partial r_{i}}{\partial \theta_{i}}\right|} .
$$

Assuming that gear 1 rotated around a fixed axis in the rigid fixed coordinate system $S_{f}$ of the frame, and gear 2 rotated around another fixed axis in $S_{q}$, the position and direction of

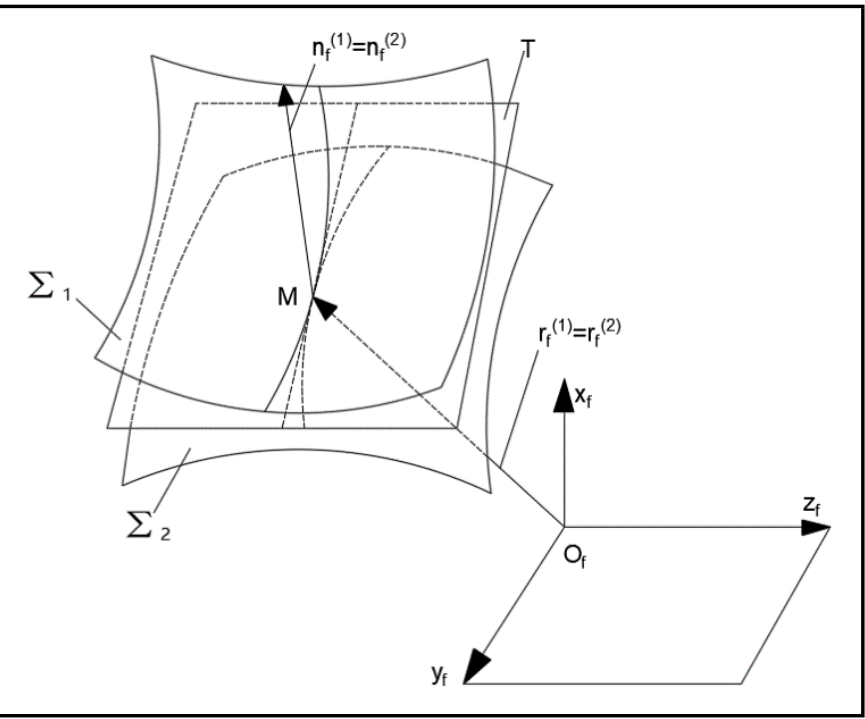

Figure 4. Contact between two tooth surfaces in ideal gear transmission.

$S_{q}$ relative to $S_{f}$ simulated the installation error, that is, the installation error was merged into gear 2 . Then the tooth surface $\Sigma_{1}$ and $\Sigma_{2}$ are expressed in $S_{f}$ as:

$$
r_{f}^{(1)}=M_{f 1} r_{1} r_{f}^{(2)}=M_{f q} M_{q 2} r_{2} .
$$

The unit normal vector was expressed in $S_{f}$ as:

$$
n_{f}^{(1)}=L_{f 1} r_{1} r_{f}^{(2)}=L_{f q} L_{q 2} n_{2} ;
$$

in the formula, the matrix $M$ was the position transformation matrix; $L$ was the vector transformation matrix.

3. Establish the meshing contact equation. The two contact surfaces must be in continuous contact, that is, their position vectors and normals were required to coincide at any instant, as shown in Fig. 4. The gear pair contact equation was:

$$
\begin{aligned}
& r_{f}^{(1)}\left(u_{1}, \theta_{1}, \varphi_{2}\right)=r_{f}^{(2)}\left(u_{2}, \theta_{2}, \varphi_{2}\right) ; \\
& n_{f}^{(1)}\left(u_{1}, \theta_{1}, \varphi_{1}\right)=n_{f}^{(2)}\left(u_{2}, \theta_{2}, \varphi_{2}\right)
\end{aligned}
$$

in the formula, $\varphi_{1}$ and $\varphi_{2}$ were the rotation angles of gear 1 and gear 2.

Three independent equations can be derived from Eq. (25), but only two independent equations can be derived from Eq. (26), because:

$$
\left|n_{f}^{(1)}\right|=\left|n_{f}^{(2)}\right|=1
$$

4. Solve the transmission error. Taking the rotation angle $\varphi_{1}$ of the driving gear 1 as the independent variable, the five unknowns and five independent quantitative equations derived from Eq. (25) and Eq. (26) were used to solve the meshing points on the tooth surface, and then the driven gear 2 The rotation angle of $\varphi_{2}^{\prime}$, and the two errors in the ideal gear transmission were solved according to Eq. (20).

\subsection{Gear Characteristic Spectrum}

Rotation frequency was related to the rotating speed of the shaft:

$$
f_{s}=\frac{N}{60}
$$




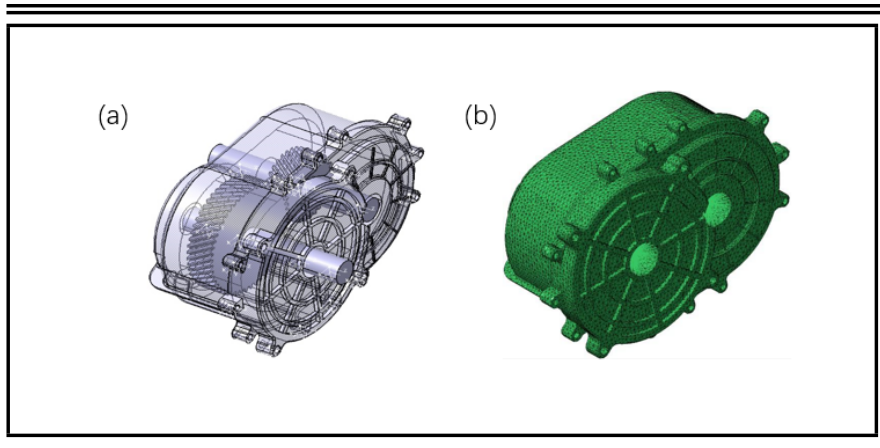

Figure 5. Gear reducer system model diagram (a) The rigid body model, (b) Rigid-flexible coupling model.

in the formula, $f_{s}$ was the frequency of rotation; $N$ was the speed of the shaft.

Gear meshing frequency, also known as impact frequency or over-tooth frequency, was produced with the meshing movement of the gear, and was equal to the number of gear teeth multiplied by the rotation frequency of the gear, which was given by:

$$
f_{m}=z f_{r}=z \frac{n}{60}
$$

in the formula, $f_{m}$ was the gear meshing frequency; $z$ was the number of gear teeth; $n$ is the gear speed.

\subsection{Sound and Power Pressure Levels}

The vibration excitation of the gear is often directly expressed in the form of sound. Therefore, the sound power level and the sound pressure level were adopted to evaluate whether the gear modification has an effect. Sound pressure refers to the change in pressure at a certain point in the fluid after being disturbed by the outside world; the sound pressure level is the ratio of the effective value of sound pressure to the effective value of reference sound pressure. Sound power refers to the total sound energy radiated by the sound source per unit time, and the sound power level is 10 times the common logarithm of the ratio of sound power to reference sound power. Current literatures mostly use the sound pressure level to calculate the sound power level (without considering the effects of temperature and atmospheric pressure): ${ }^{.25,26}$

$$
L+W=L_{P f}+10 \lg \left[\frac{S_{1}}{S_{0}}\right] ;
$$

where $L_{P f}$ is the surface sound pressure level of the measurement surface, $S_{1}$ is the area of the measurement surface, $S_{0}=1 \mathrm{~m}^{2}$.

\subsection{Rigid-flexible Coupling Dynamic Model}

According to the principle of the non-linear mathematical model of the gear pair, the LMS Virtual. Lab was used to establish a first-stage gear transmission model. As shown in Fig. 5 (a), one end of the pinion is connected to the motor as the input end, and the other end of the wheel was the output end to form a reduction mechanism. The quality attributes and inertia of the components were defined. The Bracket Joint was considered to be used to fixate the gear box with the ground, the driving shaft and the driven shaft were fixed with the gear, and the virtual input shaft was fixated with the driving shaft

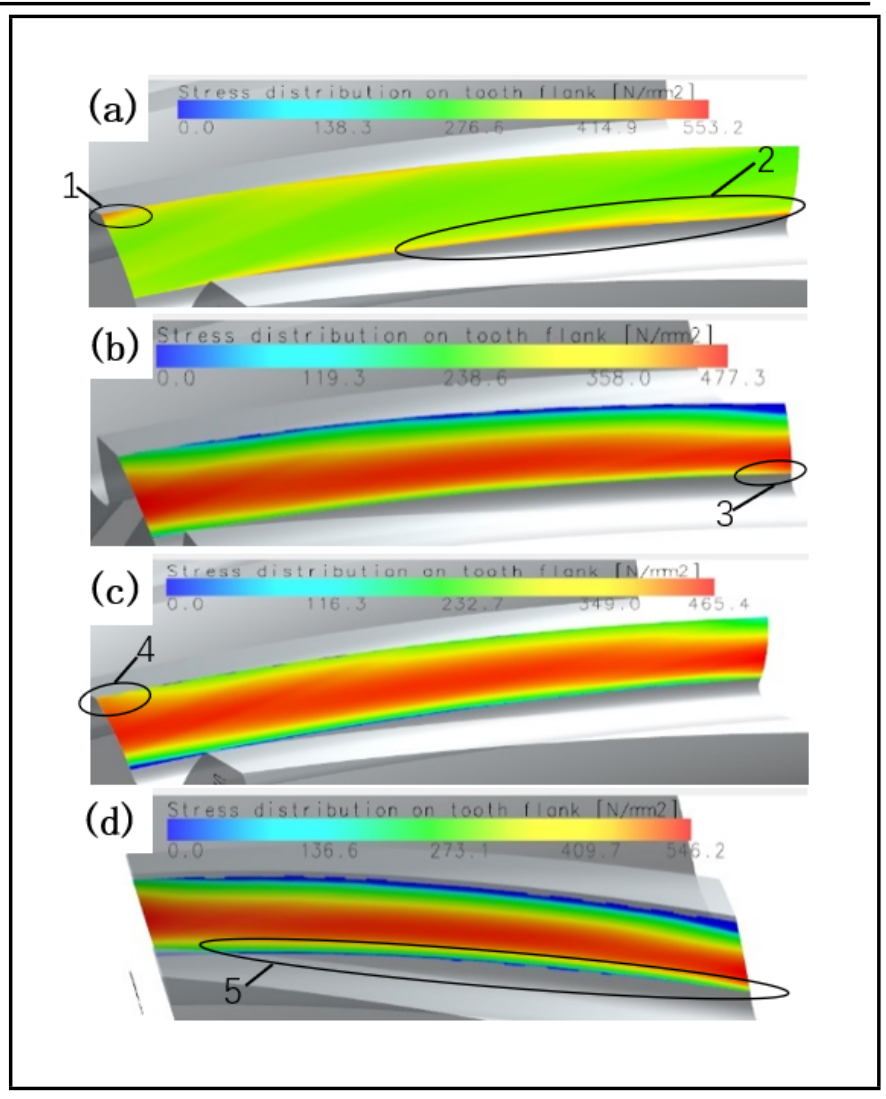

Figure 6. Simulated gear contact spots (a) Unmodified, (b) Modified pinion, (c) Modified wheel, (d) Modified wheel and pinion.

and the driven shaft was fixated with the virtual output shaft. The Revolute Joint function was used to create a rotating pair, which was designed to connect the virtual input and output axes to the earth. The Standard Bushing force was added to the bearing to simulate the effect of the bearing. Gear Contact Force was added to the Gear meshing part, the ISO standard was selected, and the influence of coincidence degree and toothed clearance was considered. Finally, the RSDA (Rotational Spring-Damper-Actuator) force was applied to the output shaft to simulate the load, and a drive was set up to rotate the input shaft. Considering that the working environment of the automobile reducer was relatively complex and different speeds were required, a speed sweep element was inserted here to scan and obtain the simulation data of $2000 \mathrm{r} / \mathrm{min}$ (low speed), $5000 \mathrm{r} / \mathrm{min}$ (medium speed) and $10000 \mathrm{r} / \mathrm{min}$ (high speed), in which the simulation step size is $0.001 \mathrm{~s}$ and the time is $2 \mathrm{~s}$.

The rigid multi-body system ignores the elastic deformation of the object in the model building process and usually be used to illustrate the low-speed operating state. Rigid-flexible coupling multi-body system refers to the coupling of a large amount of movement and elastic deformation of the object during the movement of the system. These objects need to be regarded as flexible bodies, but some objects in the system can also be regarded as rigid bodies. The rigid-flexible coupling multi-body dynamics setting of the gear transmission model established in this paper is carried out. The flexible processing was conducted in LMS Virtual. Lab with defining the mesh size of $5 \mathrm{~mm}$ as shown in Fig. 5. 


\section{RESULTS AND DISCUSSION}

\subsection{Gear Contact Spot}

When a pair of gears meshing under normal load, the contact part between the two meshing tooth surfaces forms the contact spot. The shape, size and position of the contact spots on the tooth surface directly affect the bearing capacity, service life, smooth operation and noise of the gear pair. Therefore, the tooth surface contact spot is one of the important standards of gear meshing quality. Figure 6 shows the simulation diagrams of the contact spots of wheel and pinion under different modification schemes. It can be seen that the stress concentration on the unmodified gear occurs at the edge of the tooth, that is, the root of the addendum (position 1 and 2 in Fig. 6 (a)). While the addendum and the root of the tooth are generally the weakest for gears, which is easy to cause wear, pitting and tooth breakage. After the tooth profile crowning modification of the gear, the stress is concentrated in the middle part of the gear, and the load on the tooth root part (position 3, 4, and 5 in Fig. 6) is significantly reduced. As the middle part of the gear can bear higher load than the tooth tip and root, it can effectively reduce gear failure, improve the stability and service life of the gear transmission. Compared with the maximum load $\left(553.2 \mathrm{~N} / \mathrm{mm}^{2}\right)$ on the tooth surface of unmodified gear, the load reduced by $75.9 \mathrm{~N} / \mathrm{mm}^{2}$ after modifying pinion independently, decreased by $87.8 \mathrm{~N} / \mathrm{mm}^{2}$ after modifying wheel independently, and decreased by $7 \mathrm{~N} / \mathrm{mm}^{2}$ after modifying both of the pinion and wheel. Considering the load distribution on tooth surface, after modifying both of the wheel and pinion, the load concentrated in the middle of the gear teeth, and the load at the root of the tooth tip became zero. In terms of load reduction, the maximum load reduction was obtained by modifying wheel independently.

According to the results of the simulation, the contact spot experiment was carried out on the unmodified and modified gears, and the meshing and wear conditions of the gears obtained were shown in Fig. 7 (a) and Fig. 7 (b) respectively. The results are consistent to those shown in Fig. 6 (a) and Fig. 6 (d). The severely worn positions 1 and 2 in Fig. 7 (a) have been reshaped, and the wear conditions at positions 1,2 and 3 in Fig. 7 (b) have been significantly reduced. Therefore, it can be demonstrated that the tooth profile crowning modification of the gear is effective.

\subsection{Time-varying Mesh Stiffness}

According to the simulation and experiment of gear contact spots, the contact stress of the modified gear has a better distribution than that of the unmodified gear, which may affect the performance of gear pair. Among them, the time-varying meshing force and the time-varying meshing stiffness are the most important factors to reflect the gear meshing effect. The ISO method was used to carry out the dynamic simulation of rigid-flexible coupling of the gear pair. Fig. 8 shows the time-varying meshing stiffness curves of the helical gear pair before and after tooth profile crowning modification. It can be found that the time-varying meshing stiffness is the lowest after modifying the tooth profile crowning shape of wheel and pinion simultaneously. The fluctuation range is reduced to
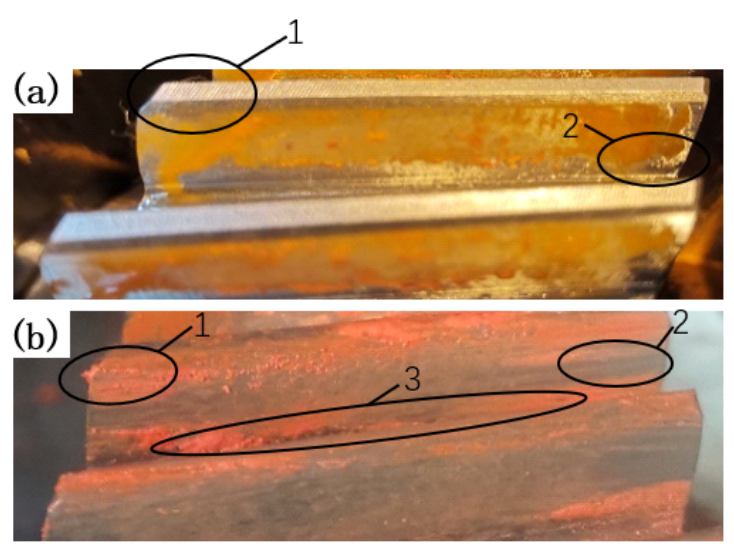

Figure 7. Experimental results of contact spots (a) Contact spots of unmodified wheel, (b) Contact spots of modified wheel.

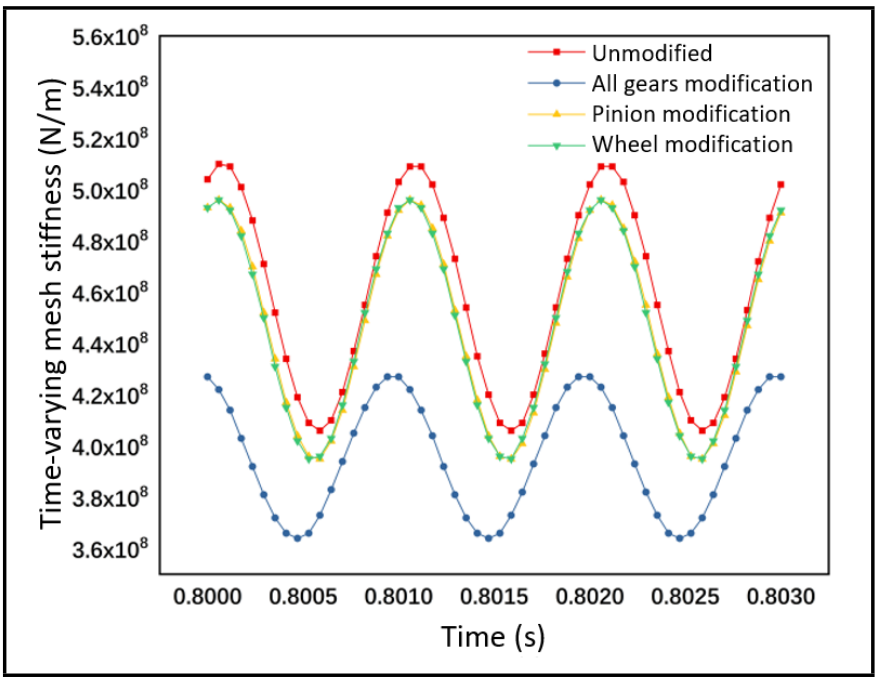

Figure 8. Time-varying meshing stiffness before and after the tooth profile crowning shape modification.

$3.64 \times 108 \sim 4.27 \times 108 \mathrm{~N} / \mathrm{m}$, and the peak value drops by $16.3 \%$ in comparison with that of unmodified gear pair. But if the pinion or wheel is independently modified by tooth profile crowning, the time-varying meshing stiffness is almost the same, the peak value drops to $4.96 \times 108 \mathrm{~N} / \mathrm{m}$ with the dropping rate of $2.7 \%$. The reason could be that the modification quantity $3 \mu \mathrm{m}$ of the gear pair is selected when the Hertz contact stress and transfer error reach the minimum value at the same time, which attributes to the reduction of time-varying meshing stiffness.

Figure 9 is the time-varying meshing force curve of the helical gear pair before and after of the tooth profile crowning modification under $2000 \mathrm{r} / \mathrm{min}, 5000 \mathrm{r} / \mathrm{min}$, and $10000 \mathrm{r} / \mathrm{min}$, respectively. If the tooth profile crowning modification is performed on the wheel and pinion at the same time or the single wheel, not only the time-varying meshing force of the helical gear pair is greatly reduced, but also the meshing impact phenomenon disappears. After tooth profile crowning modifying the helical gear pair of an electric vehicle reducer box, the peak time-varying meshing force at low speed, medium speed and high speed decreased by $172.484 \mathrm{~N}, 1989.976 \mathrm{~N}$, and $2936.359 \mathrm{~N}$, respectively, in comparison with that of the unmodified gear pair. However, if only the pinion is modified, 


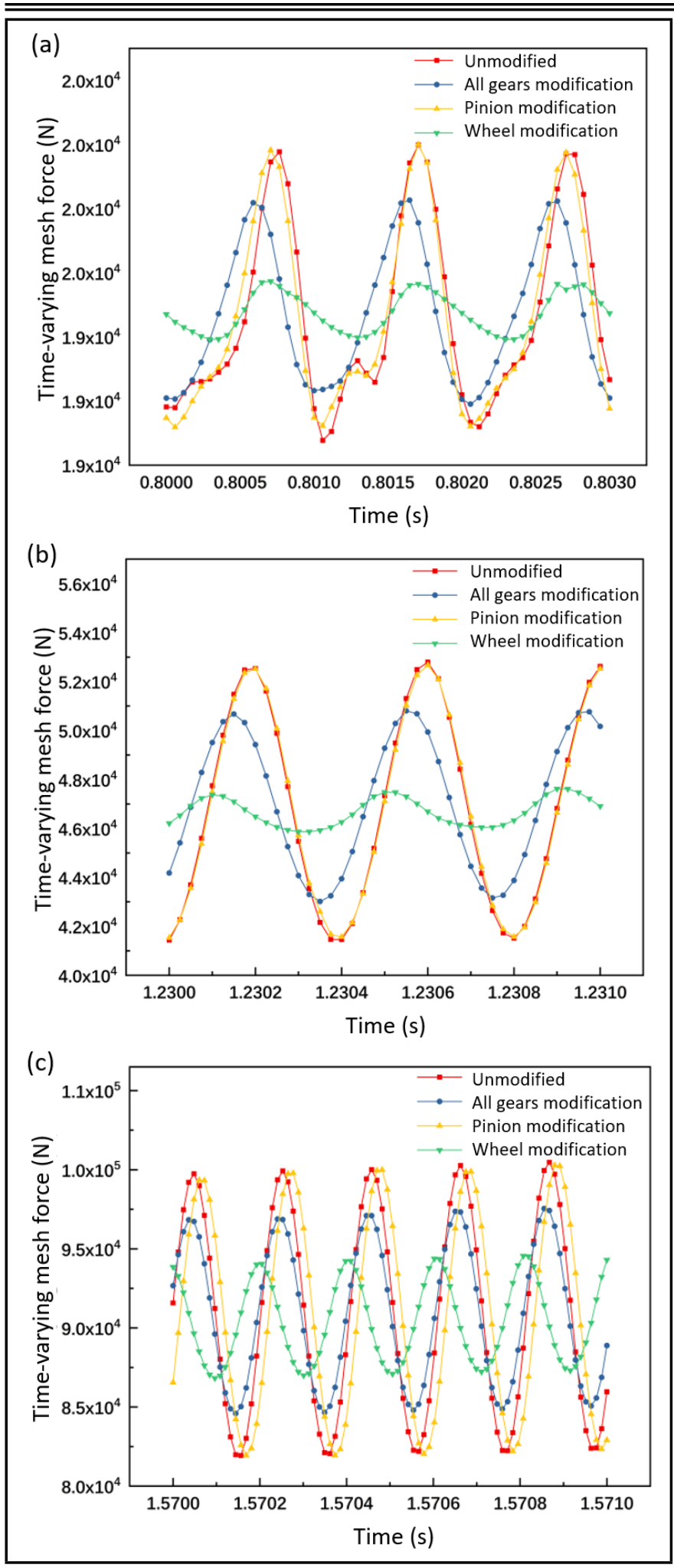

Figure 9. Comparison of time-varying meshing force before and after tooth profile crowning modification (a) $2000 \mathrm{r} / \mathrm{min}$, (b) $5000 \mathrm{r} / \mathrm{min}$, (c) $10000 \mathrm{r} / \mathrm{min}$.

the time-varying meshing force of the gear cannot be reduced, and the meshing impact phenomenon is still serious. Compared with the other two tooth profile crowning optimization schemes, the positive effect is unavailable. Therefore, it is not appropriate to modifying the pinion independently. For the optimization of the tooth profile crowning modification method, the selection of the modified gear is critical. If the main purpose is to reduce the rigidity excitation of the helical gear pair, it is recommended to modify the wheel and pinion at the same
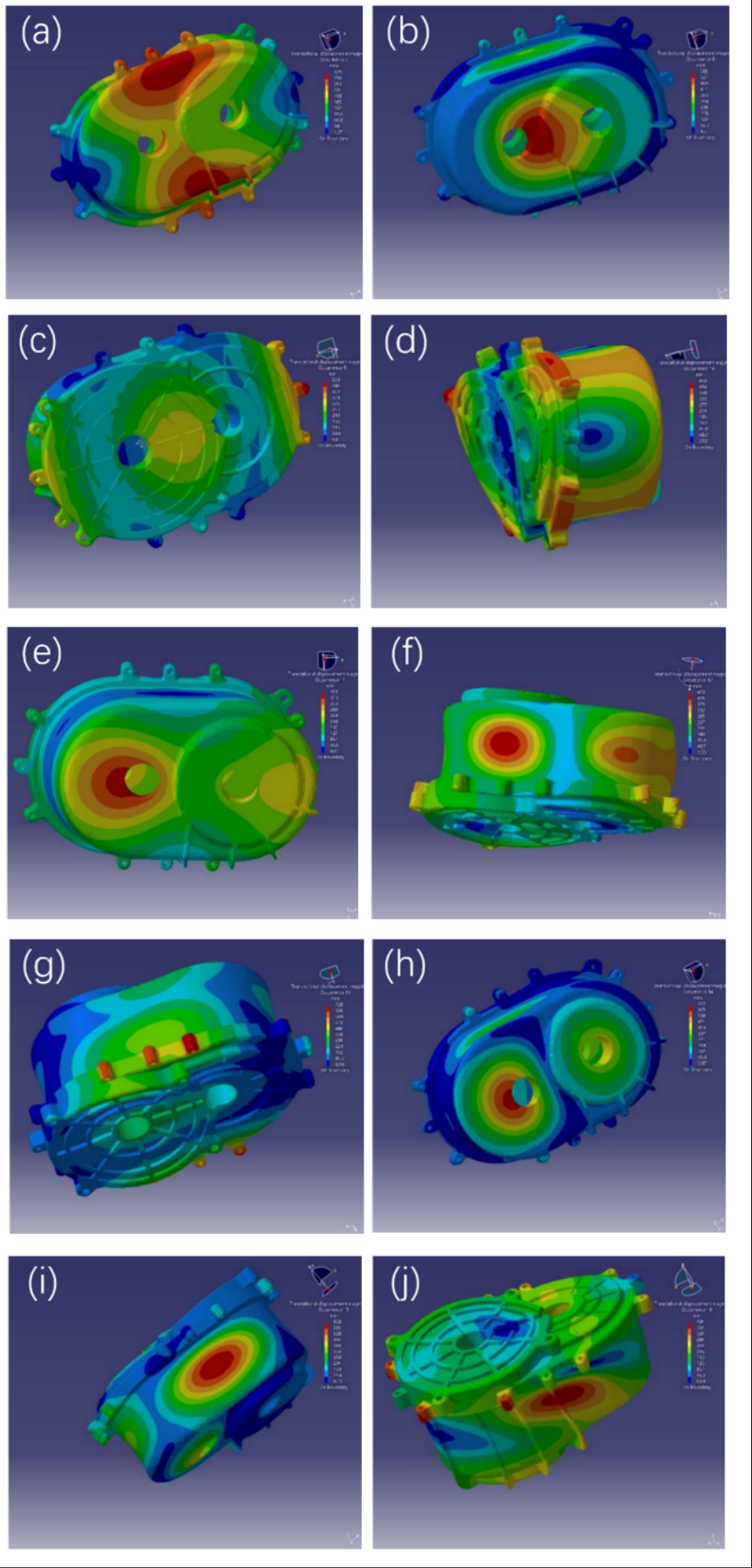

Figure 10. Modal response of the first to tenth order (a) the first mode, (b) the second mode, (c) the third mode, (d) the Fourth mode, (e) the fifth mode, (f) the sixth mode, (g) the seventh mode, (h) the eighth mode, (i) The ninth mode, (j) the tenth mode.

time. If the goal is to reduce the time-varying meshing force of the helical gear pair, eliminating the meshing impact and preventing the gear pair from violent vibration, it is recommended to modify the wheel or modify wheel and pinion together.

\subsection{Modal Analysis}

Vibration modes are inherent and integral characteristics of elastic structures. Through the modal analysis method, the characteristics of the main modes of the gearbox in a certain susceptible frequency range can be clarified. Based on the characteristics of these modes, the actual vibration response generated by various external or internal vibration sources in 


Table 3. Modal response parameters of the first to tenth order.
\begin{tabular}{||c|c|c|}
\hline Order & Natural frequency/Hz & Mode shape \\
\hline 1 & 3823.25 & Longitudinal bending \\
\hline 2 & 4105.95 & Axial bending \\
\hline 3 & 4913.10 & Lateral bending \\
\hline 4 & 5565.12 & Longitudinal twist \\
\hline 5 & 5664.21 & Lateral bending \\
\hline 6 & 7103.24 & Longitudinal bending \\
\hline 7 & 7478.99 & Axial bending \\
\hline 8 & 8062.73 & Axial torsion \\
\hline 9 & 8425.08 & Vertical expansion \\
\hline 10 & 8520.22 & Longitudinal twist \\
\hline
\end{tabular}

this frequency band of the gearbox could be clarified. In this research, the advanced Nastran Craig-Bampton modal analysis method was adopted, and the calculation was performed after setting the frequency domain response range.

The results are shown in Fig. 10. It can be seen that the main vibration modes of the box in the first 10 orders are torsional or bending vibrations along the transverse, longitudinal and axial directions. The modal natural frequencies of first to tenth order are displayed in Table 3. Since the natural frequency of the first order mode reached $3823.25 \mathrm{~Hz}$, its low-frequency vibration characteristics are better. Considering that if the modal natural frequency of the shell is close to the meshing frequency of the gear pair in the box, it may tend to cause resonance. Generally, it is suggested that the natural frequency of the box should be kept away from the "resonant frequency" by setting stiffeners, drilling holes and changing the wall thickness in engineering practice. As the vibration characteristics of box body were good at low and medium speed, and no resonance phenomenon occurred at high speed, it is conducive to clarifying the gear modification effects on the vibration and noise of the reducer after excluding the influence of housing.

\subsection{Vibration and Noise}

Through calculation by LMS Virtual. Lab simulation, the sound power levels are obtained under different working conditions. As shown in Fig. 11, although the peak sound power does not reduce at the meshing frequency under high-speed conditions when the pinion is modified, its average sound power value is only $56.9 \mathrm{~dB}(\mathrm{~A})$, decreasing by $1.5 \mathrm{~dB}(\mathrm{~A})$, and the goal of noise reduction in almost the entire frequency domain is achieved. However, for medium-speed or lowspeed conditions, the noise reduction effect of this modification scheme is not ideal, especially for low-speed conditions, the high-frequency $(4978.5 \sim 8525 \mathrm{~Hz}$ ) noise increases significantly.

Due to the optimization scheme, the time-varying meshing stiffness (stiffness excitation) and transmission error (error excitation) of the modified helical gear pair are reduced, and the time-varying meshing force of the gear at this time shows no obvious change. Normally, the dynamic excitation of the modified helical gear pair tends to decrease because of the decreasing stiffness excitation and error excitation, so that the noise power will decline. However, it increases significantly at low and medium speeds within a relative high frequency domain. This may be related to the fact that modifying the crowning shape of the pinion independently will aggravate the frequency and degree of meshing impact between the helical gear pairs,
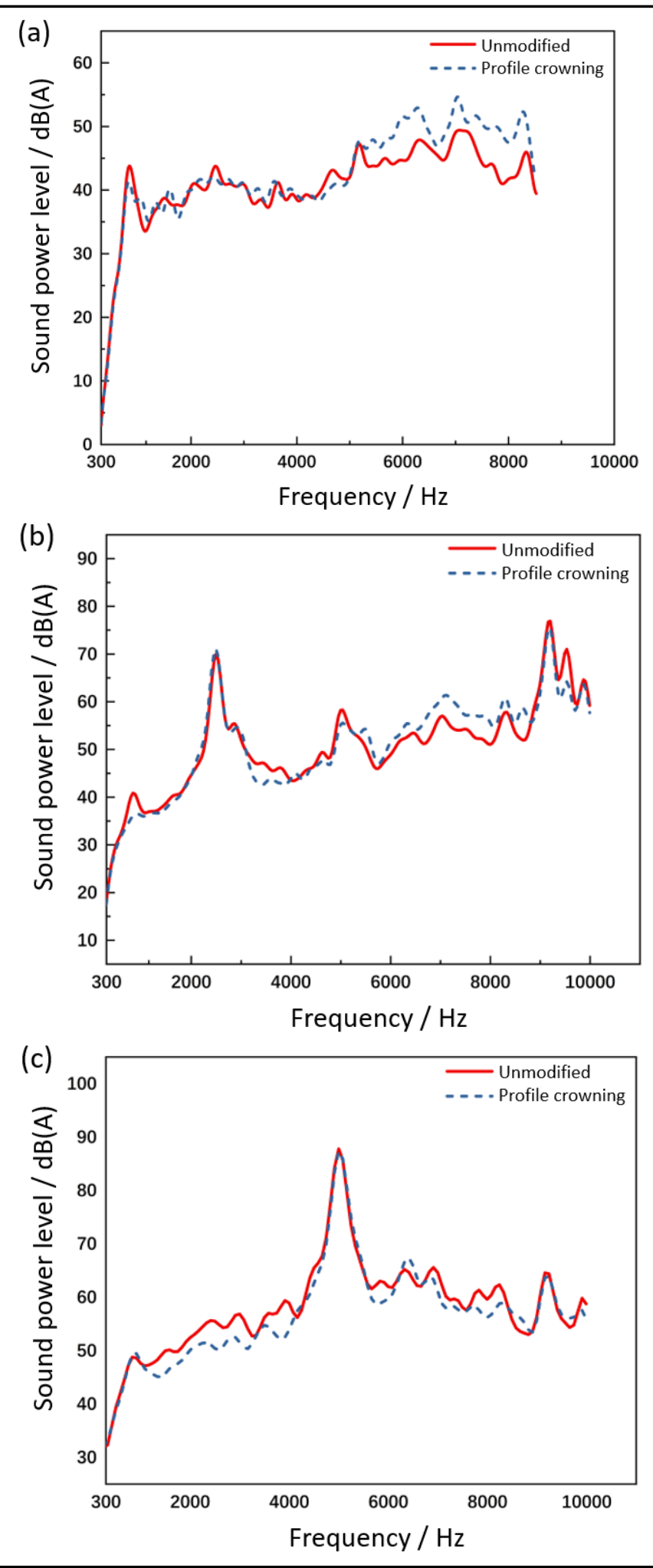

Figure 11. Sound power levels of different speeds under pinion modification (a) $2000 \mathrm{r} / \mathrm{min}$, (b) $5000 \mathrm{r} / \mathrm{min}$, (c) $10000 \mathrm{r} / \mathrm{min}$

making the transmission not smooth; or it is due to the failure in reducing time-varying meshing force of the helical gear at this speed, where the dynamic excitation inside mainly depends on the time-varying meshing force.

As can be seen from Fig. 12, the overall noise reduction effect is ideal when the tooth profile crowning modification is adopted for the wheel. The noise reduction in the full frequency domain is almost achieved under low-speed conditions, 


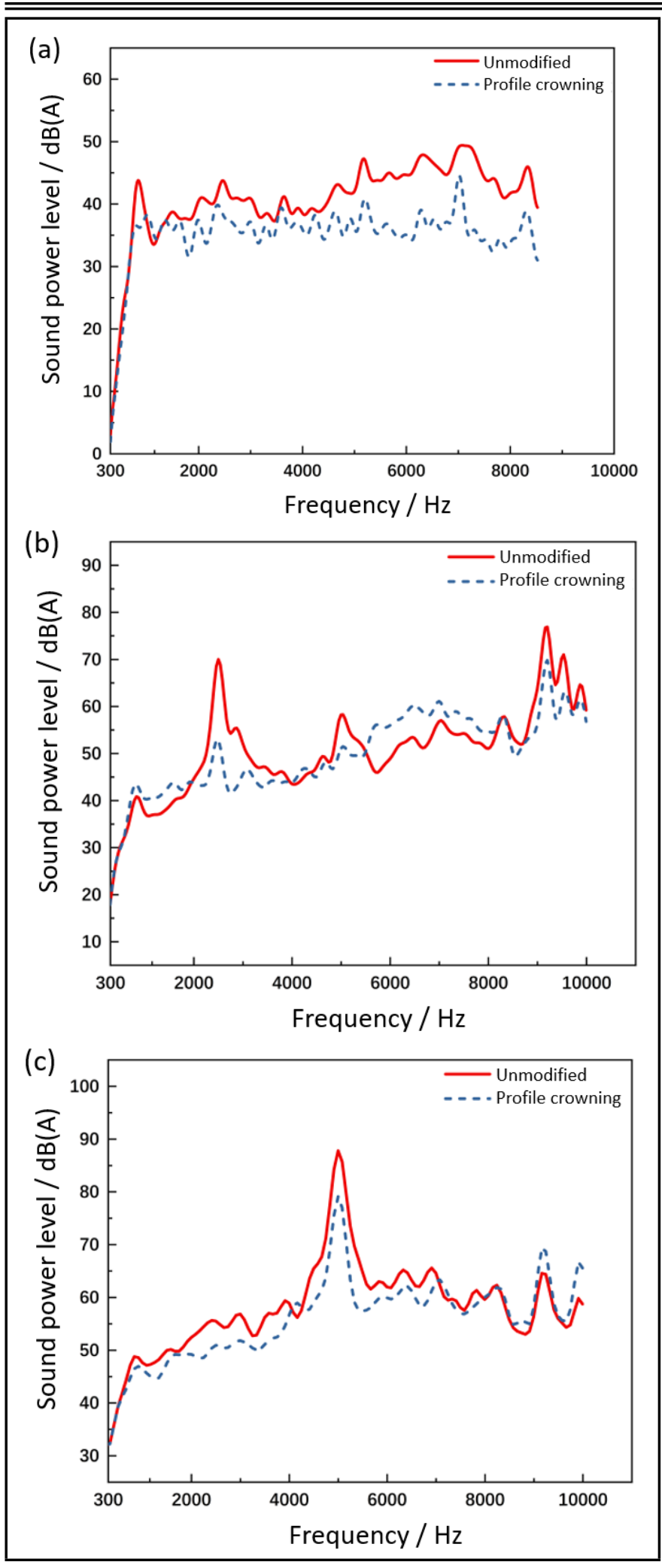

Figure 12. Sound power levels of different speeds under wheel modification (a) $2000 \mathrm{r} / \mathrm{min}$, (b) $5000 \mathrm{r} / \mathrm{min}$, (c) $10000 \mathrm{r} / \mathrm{min}$.

and the peak sound power at the meshing frequency and the average sound power at this speed have changed from $43.7 \mathrm{~dB}(\mathrm{~A})$ and $40.9 \mathrm{~dB}(\mathrm{~A})$ to $36.3 \mathrm{~dB}(\mathrm{~A})$ and $35.4 \mathrm{~dB}(\mathrm{~A})$ after modifying wheel, with decreasing rate of $16.9 \%$ and $13.4 \%$, respectively. In addition, the modifying scheme achieves most of the frequency domain noise reduction under medium and high-speed conditions. The peak sound power values at the meshing frequency drop from $70 \mathrm{~dB}(\mathrm{~A})$ and $87.8 \mathrm{~dB}(\mathrm{~A})$ to $52.9 \mathrm{~dB}(\mathrm{~A})$ and
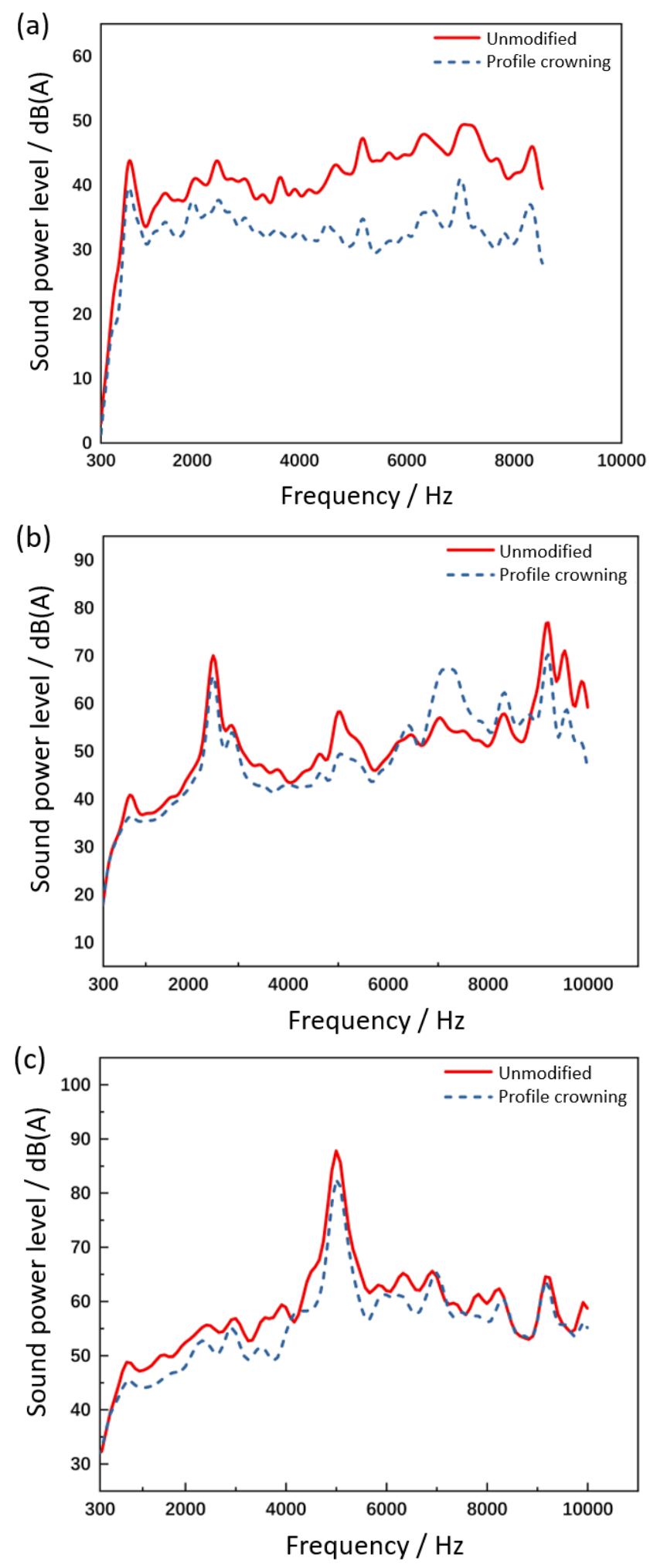

Figure 13. Sound power levels of different speeds under modification of wheel and pinion (a) $2000 \mathrm{r} / \mathrm{min}$, (b) $5000 \mathrm{r} / \mathrm{min}$, (c) $10000 \mathrm{r} / \mathrm{min}$.

$79.1 \mathrm{~dB}(\mathrm{~A})$, while the average sound power values drop from $50.9 \mathrm{~dB}(\mathrm{~A})$ and $58.4 \mathrm{~dB}(\mathrm{~A})$ to $50.3 \mathrm{~dB}(\mathrm{~A})$ and $56.1 \mathrm{~dB}(\mathrm{~A})$, respectively. This is because the transmission error, time-varying meshing stiffness, and time-varying meshing force of the helical gear pair modified by this scheme are effectively reduced. At the same time, the gear time-varying meshing force curve is smoother, and the meshing impact is greatly improved.

As can be seen from Fig. 13, the sound power of the he- 
lical gear pair decreases significantly after the tooth profile crowning modification of the wheel and pinion under the three working conditions. Especially in low-speed and high-speed working conditions, full frequency domain noise reduction is achieved. Even in medium speed working conditions where the noise reducing effect is relatively poor, noise reduction within most of the frequency domain except for $6202.3 \sim$ $8824.7 \mathrm{~Hz}$ has been achieved. The peak sound power of the modified helical gear pair at meshing frequencies under lowspeed, medium-speed, and high-speed working conditions is only $39.6 \mathrm{~dB}(\mathrm{~A}), 65.8 \mathrm{~dB}(\mathrm{~A})$, and $82.5 \mathrm{~dB}(\mathrm{~A})$, which is reduced by $4.1 \mathrm{~dB}(\mathrm{~A}), 4.2 \mathrm{~dB}(\mathrm{~A})$, and $5.3 \mathrm{~dB}(\mathrm{~A})$ with the decreasing rate of $9.4 \%, 6.0 \%, 6.0 \%$, respectively. Similarly, the average sound power values of the modified helical gear pair under different working conditions have also dropped to $32.5 \mathrm{~dB}(\mathrm{~A}), 49.2 \mathrm{~dB}(\mathrm{~A})$, and $55.4 \mathrm{~dB}(\mathrm{~A})$, which are reduced by $8.4 \mathrm{~dB}(\mathrm{~A}), 1.7 \mathrm{~dB}(\mathrm{~A})$, and $3 \mathrm{~dB}(\mathrm{~A})$, respectively. As the sound power is reduced by $3 \mathrm{~dB}(\mathrm{~A})$, the energy becomes half of the original value, which means this modification scheme has an excellent vibration damping effect at low and high speeds. At the same time, according to the contact spot and dynamic analysis, the transmission error, time-varying meshing force, and time-varying meshing stiffness of the helical gear pair after optimizing and modifying with this scheme are all reduced. This indicates that the modified helical gear pair has less internal excitation, and its vibration and noise will naturally become lower.

Previous investigations on the gear modification of powertrain system presented valuable results, especially the simulation research conducted by using MASTA software. According to the contact damage rate and safety factor, a similar result was drawn that reasonable shape modification could reduce the vibration and noise of transmission system under the actual working condition. ${ }^{27}$ Combining with the experiment of gear contact spot, the gear contact pressure could be evaluated. By multi-objective optimization, it was found that the contact pressure, transmission error and noise of modified gear can be significantly reduced. ${ }^{28}$ In addition, tooth profile modification is conducive for planetary gear system by reducing the sudden change of tooth flank load during gear meshing. When the internal and external meshing pairs alternate between single and double teeth, tooth profile modification can effectively restrain the sudden change of meshing stiffness, and reduce the fluctuation of transmission error. ${ }^{29}$ Moreover, the selection of the modified gear is extremely critical. For example, modifying the wheel independently or both of the pinion and wheel have good vibration and noise reduction effects. However, if only the pinion is modified, the overall noise sound power level will increase although its transmission error and time-varying meshing stiffness are reduced. Considering the processing and manufacturing costs, modifying the wheel independently is recommended in practice.

\section{EXPERIMENTAL VERIFICATION}

The bench test was carried out in the semi-muffled noise laboratory. The test bench is shown in Fig. 14, and the ninepoint sound pressure measurement method is adopted to measure the noise. According to the simulation results of vibra-

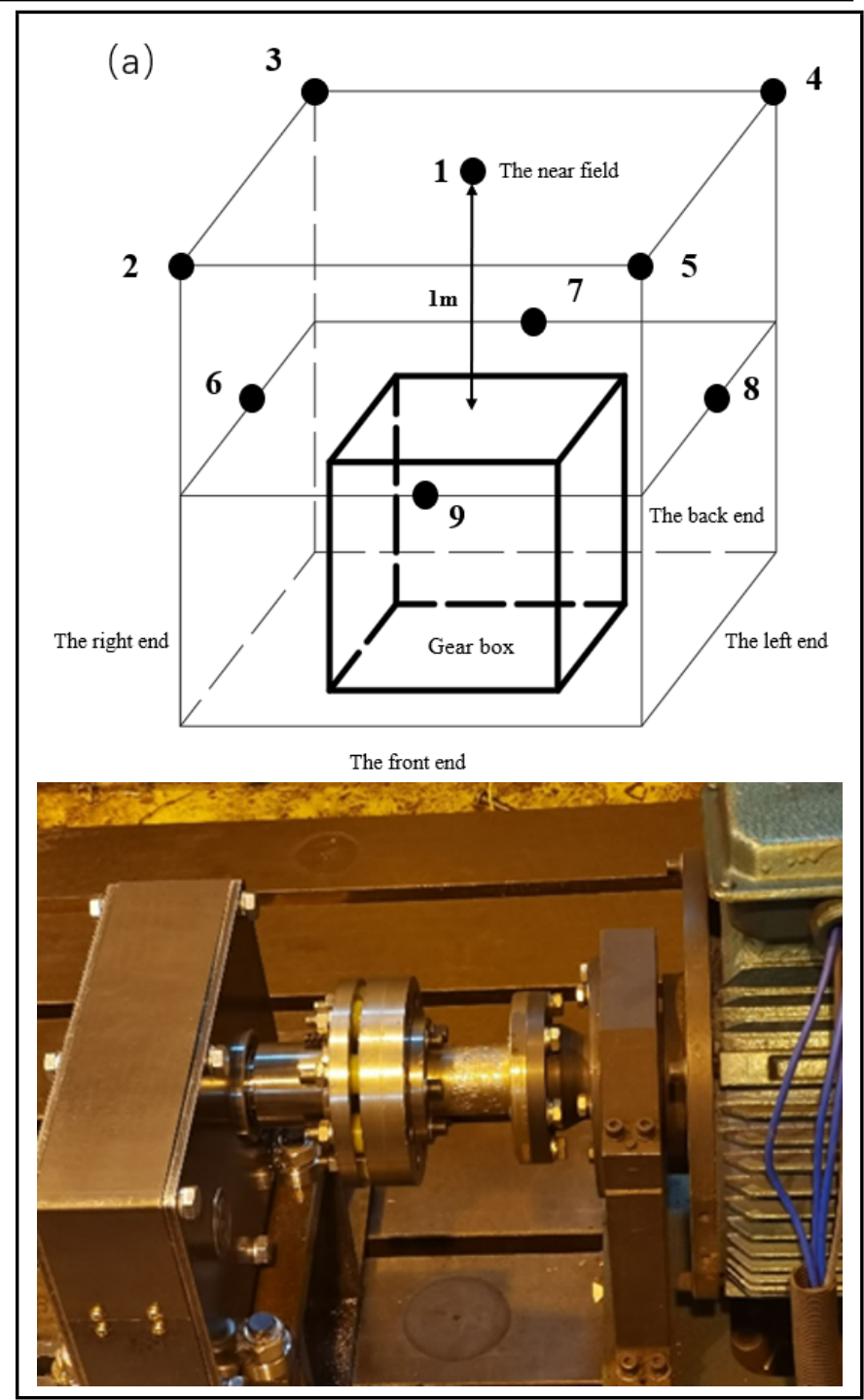

Figure 14. (a) Diagram of nine-point sound pressure measurement, (b) Vibration and noise test bench.

tion and noise, the scheme for modifying both the wheel and pinion is selected for experimental verification. The gear box used in the experiment adopts a simple rectangular shape, and the basic parameters of the gear is consistent with those used in simulation as shown in Table 1. The test bench is shown in Fig. 14, and the nine-point sound pressure measurement method is adopted to measure the noise.

According to the measured sound pressure value, the sound power is calculated as shown in Fig. 15. By comparing the noise of the gearbox before and after the modification obtained in the experiment. It can be seen that the values of sound power levels measured at the same points tend to be similar before and after modification. From the point of noise reduction, the noise at the 8th point decreases the most, from $76.9 \mathrm{~dB}(\mathrm{~A})$ to $72.9 \mathrm{~dB}(\mathrm{~A})$, which is similar to the noise reduction obtained in simulation. Therefore, this demonstrates that the accuracy of the simulation results can be achieved, also proves that the acoustic simulation ideas provided in this article are reasonable and effective. However, it should be noted that due to being limited by the experimental conditions, the gearbox used in this experiment has a different structure from the gearbox in the simulation, and the noise of the motor used in the ex- 


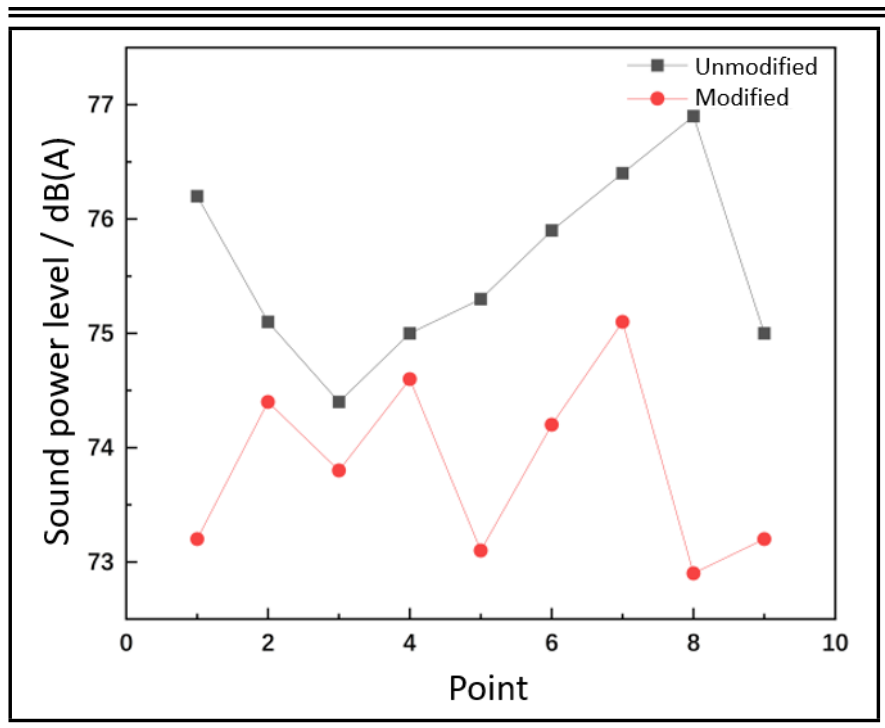

Figure 15. Sound power level of the nine-point measurement method.

periment causes the measured noise value to be larger during the experiment, which has impacts on the experimental results. In summary, the experimental results validate that appropriate tooth profile crowning modification is conducive to controlling the gear meshing vibration and noise of reducer.

\section{CONCLUSIONS}

Based on the transmission model of the electric vehicle reducer, the tooth profile crowning modification is adopted on a pair of gears to reduce vibration and noise. The contact spots of gears under different modification schemes are compared with the experimental results. The rigid-flexible coupling multibody dynamics analysis method is used to simulate the dynamics and acoustics, and the time-varying meshing stiffness and force, the vibration and noise under typical working conditions were compared and analyzed. The following conclusions are drawn:

For the tooth profile crowning modification, the selection of modified gear is very important. To reduce the rigidity excitation of the helical gear pair, it is recommended that the wheel and pinion be modified at the same time; to reduce the timevarying meshing force and meshing shock, and prevent the gear pair from violent vibration, it is recommended to modify the wheel or both of the pinion and wheel, while modifying the pinion independently is not suitable.

According to the comparison of contact spots, the concentration of stress occurs at the tooth tip and root of unmodified gear, while it occurs at the middle position of the gear flank after modification, which effectively reduces the wear load and internal excitation of the gear pair.

Although the average sound power level is reduced by $1.5 \mathrm{~dB}(\mathrm{~A})$, modifying the pinion independently cannot reduce the time-varying meshing stiffness and force during the gear transmission process, the sound power level rises significantly under low-speed and high-frequency working conditions.

Modifying the wheel independently or both the pinion and wheel have advantages in reducing vibration and noise and modifying both the pinion and wheel is slightly better. Considering the processing and manufacturing costs, modifying the wheel independently is recommended in practice.

\section{ACKNOWLEDGEMENTS}

This work was funded by the National Natural Science Foundation of China (Grant number 51975252) and the Postgraduate Research \& Practice Innovation Program of Jiangsu Province (Grant number KYCX19_1597).

\section{REFERENCES}

1 Tanaka, S., Ezoe, S., and Ide, K. Appreciable improvements in oil film formation and surface durability of gears with tooth profile modification. JSME international journal. Ser. 3, Vibration, control engineering, engineering for industry, 31(2), 431-435, (1988). https://dx.doi.org/10.1299/jsmec1988.31.431

2 Fatourehchi, E., Mohammadpour, M., King, P. D., Rahnejat, H., and Trimmer, G. Effect of tooth profile modification on the durability of planetary hub gears. International Journal of Powertrains, 8(1), 40-57, (2019). https://dx.doi.org/10.1504/IJPT.2019.098120

3 Wang, C. Study on 3-D modification for reducing vibration of helical gear based on TCA technology, LTCA technology and system dynamics. Mechanical Systems and Signal Processing, 146, 106991, (2021). https://dx.doi.org/10.1016/j.ymssp.2020.106991

4 Yang, R., Han, B. and Xiang, J. Nonlinear Dynamic Analysis of a Trochoid Cam Gear with the Tooth Profile Modification. Int. J. Precis. Eng. Manuf. 21, 2299-2321 (2020). https://dx.doi.org/10.1007/s12541-020-00417-6

5 Samani, F. S., Molaie, M., and Pellicano, F. Nonlinear vibration of the spiral bevel gear with a novel tooth surface modification method. Meccanica, 54(7), 1071-1081, (2019). https://dx.doi.org/10.1007/s11012-019-00973-w

6 Ren, Z. Y., Mao, S. M., Guo, W. C., and Guo, Z. Tooth modification and dynamic performance of the cycloidal drive. Mechanical Systems and Signal Processing, 85, 857-866, (2017). https://dx.doi.org/10.1016/j.ymssp.2016.09.029

7 Brethee, K. F., Gu, F., and Ball, A. D. Frictional effects on the dynamic responses of gear systems and the diagnostics of tooth breakages. Systems Science \& Control Engineering, 4(1), 270-284, (2016). https://dx.doi.org/10.1080/21642583.2016.1241728

8 Hjelm, R., Ahadi, A., and Wahlström, J. Gear tolerancing for simultaneous optimization of transmission error and contact pressure. Results in Engineering, 9, 100195, (2021). https://dx.doi.org/10.1016/j.rineng.2020.100195

9 Wang, Y. N., Sun, Z. L., and Yin, M. A. Considering thermal deformation in gear transmission error calculation, Applied Mechanics and Materials, 281, 211-215, (2013). https://dx.doi.org/10.4028/www.scientific.net/AMM.281.211

10 Duan, T., Wei, J., Zhang, A., Xu, Z., and Lim, T. C. Transmission error investigation of gearbox using rigid-flexible coupling dynamic model: 
Theoretical analysis and experiments. Mechanism and Machine Theory, 157, 104213, (2021). https://dx.doi.org/10.1016/j.mechmachtheory.2020.104213

11 Slamani, M., and Bonev, I. A. Characterization and experimental evaluation of gear transmission errors in an industrial robot. Industrial Robot: An International Journal, 40(5), 441-449, (2013). https://dx.doi.org/10.1108/IR-072012-387

12 Wink, C. H., and Serpa, A. L. Investigation of tooth contact deviations from the plane of action and their effects on gear transmission error. Proceedings of the Institution of Mechanical Engineers, Part C: Journal of Mechanical Engineering Science, 219(5), 501-509, (2005). https://dx.doi.org/10.1243/095440605X16983

13 Palermo, A., Britte, L., Janssens, K., Mundo, D., and Desmet, W. The measurement of gear transmission error as an NVH indicator: theoretical discussion and industrial application via low-cost digital encoders to an all-electric vehicle gearbox. Mechanical Systems and Signal Processing, 110, 368-389, (2018). https://dx.doi.org/10.1016/j.ymssp.2018.03.005

14 El Yousfi, B., Soualhi, A., Medjaher, K., and Guillet, F. New approach for gear mesh stiffness evaluation of spur gears with surface defects. Engineering Failure Analysis, 116, 104740, (2020). https://dx.doi.org/10.1016/j.engfailanal.2020.104740

15 Dai, H., Long, X., Chen, F., and Xun, C. An improved analytical model for gear mesh stiffness calculation. Mechanism and Machine Theory, 159, (2021). https://dx.doi.org/10.1016/j.mechmachtheory.2021.104262

16 Chen, K., Huangfu, Y., Ma, H., Xu, Z., Li, X., and Wen, B. Calculation of mesh stiffness of spur gears considering complex foundation types and crack propagation paths. Mechanical Systems and Signal Processing, 130, 273-292, (2019). https://dx.doi.org/10.1016/j.ymssp.2019.05.014

17 Raghuwanshi, N. K., and Parey, A. A new technique of gear mesh stiffness measurement using experimental modal analysis. Journal of Vibration and Acoustics, 141(2), 021018, (2019). https://dx.doi.org/10.1115/1.4042100

18 Chen, Z., Zhou, Z., Zhai, W., and Wang, K. Improved analytical calculation model of spur gear mesh excitations with tooth profile deviations. Mechanism and Machine Theory, 149, 103838, (2020). doi: https://dx.doi.org/10.1016/j.mechmachtheory.2020.103838

19 Tang, Z. P., Sun, J. P., Yan, L., and Lou, F. Dynamic Contact Analysis And Tooth Modification Design For Emu Traction Gear. International Journal of Simulation Modelling, 16(4), (2017). https://dx.doi.org/10.2507/IJSIMM16(4)CO20

${ }^{20}$ Liu, Y. Y., Xiong, M. C., and Li, Q. High Speed Gear Parametric Modeling and Dynamic Analysis, Advanced Materials Research, 945, 849-852, (2014).
https://dx.doi.org/10.4028/www.scientific.net/AMR.945949.849

21 Jia, H., Qin, D., and Liu, C. Novel tooth modification methodology for multistage spur gears considering dynamic deformation of housing. Proceedings of the Institution of Mechanical Engineers, Part C: Journal of Mechanical Engineering Science, 233(21-22), 7257-7269, (2019). https://dx.doi.org/10.1177/0954406219839096

22 Jun, Z., Wei-min, T., Qin, C., and Tao, C. Reliability sensitivity analysis of tooth modification on dynamic transmission error of helical planetary gears. Proceedings of the Institution of Mechanical Engineers, Part C: Journal of Mechanical Engineering Science, 234(19), 3903-3918, (2020). https://dx.doi.org/10.1177/0954406220917424

23 Fang, J., Xiao, J., Li, L., and Zhang, D. On dynamics of a rotating hub-flexible rod-concentrated mass system considering rigid-flexible coupling effect. Journal of Vibroengineering, 22(5), 1006-1021, (2020). https://dx.doi.org/10.21595/jve.2020.21088

24 Mark, W. D. Time-synchronous-averaging of gearmeshing-vibration transducer responses for elimination of harmonic contributions from the mating gear and the gear pair. Mechanical Systems and Signal Processing, 62, 21-29, (2015). https://dx.doi.org/10.1016/j.ymssp.2015.03.006

25 Campillo-Davo, N., Peral-Orts, R., Campello-Vicente, H., and Velasco-Sanchez, E. An alternative closeproximity test to evaluate sound power level emitted by a rolling tyre. Applied Acoustics, 143, 7-18, (2019). https://dx.doi.org/10.1016/j.apacoust.2018.08.017

26 Campillo-Davo, N., Peral-Orts, R., Campello-Vicente, H., and Velasco-Sanchez, E. A methodology for the extrapolation of coast-by noise of tyres from sound power level measurements. Applied Acoustics, 159, (2020). https://dx.doi.org/10.1016/j.apacoust.2019.107077

$27 \mathrm{Li}$ Y X, Shen W Q, Zhao Y J, et al. Gear modification analysis to improve the vibration and noise of automobile transmission[C]. Key Engineering Materials, 579, 265-269, (2014). https://dx.doi.org/10.4028/www.scientific.net/KEM.579580.265

28 Liu J, Xiong F, Wei D, et al. Research of Gear Modification on Noise Optimization of EV Reducer[C]. Journal of Physics: Conference Series, 1802(4), 042090, (2021). https://dx.doi.org/10.1088/1742-6596/1802/4/042090

$29 \mathrm{Xu}$ X, Fan X, Diao P, et al. An investigation on the influence of modification parameters on transmission characteristics of planetary gear system[J]. Journal of Mechanical Science and Technology, 33(7), 3105-3114, (2019). https://dx.doi.org/10.1007/s12206-019-0605-6 